\title{
10th Rib Cartilage: Another Option of the Costal Cartilage Graft for Rhinoplasty
}

\author{
Jae Hoon Kim, Jin Woo Song, \\ Sung Wan Park, Won Suk Oh, \\ Joo Heon Lee
}

April 31 Plastic Surgery Clinic, Seoul, Korea

No potential conflict of interest relevant to this article was reported.
Background The costal cartilage allows for versatile and stable cartilage supply for rhinoplasty but disadvantages remain; to minimize these, the 10th costal cartilage was used. We present our experience with the use of the 10th costal cartilage as autologous graft material for rhinoplasty.

Methods Sixty-four patients whose 10th costal cartilage had been used for correction of severe caudal nasal deviation, secondary cleft lip nasal deformity, and secondary rhinoplasty with difficulty using the septal and auricular cartilages were enrolled in this study from November 2008 to December 2012. To evaluate the efficacy and safety of this method in rhinoplasty, donor-site morbidity, scarring, and postoperative results, including availability of graft material and complications, were assessed.

Results The mean time for cartilage harvesting was 18 minutes, and the mean length of the harvested cartilage was $2.4 \mathrm{~cm}$. The harvested cartilage was used for columellar struts and tip onlay grafts. The natural curvature of the 10th costal cartilage was used, depending on the purpose of the strut. It could be applied without carving in 38 cases (59\%). Although revision was performed in three patients, there were no graft-related complications.

Conclusions The 10th costal cartilage can be harvested safely and quickly, and its handling procedure is simple. As such, it is a good option as an autologous graft for rhinoplasty.

Keywords Rhinoplasty, Costal cartilage, Ribs

\section{INTRODUCTION}

The septal and auricular cartilages have traditionally been considered primary autologous graft materials for rhinoplasty [1-6]. However, the autologous costal cartilage can be useful when a strong strut is required due to contracture from previous surgeries or accidents, when tip projection is required, when the septal and auricular cartilage cannot be used due to a previous surgery, or when a large amount of autologous cartilage is needed [7-12].

Received: Feb 14, 2015 Revised: Apr 14, 2015 Accepted: Apr 14, 2015 Correspondence: Jae Hoon Kim April 31 Plastic Surgery Clinic, 6th floor, Geonwoo B/D, 548 Gangnam-daero, Gangnam-gu, Seoul 135-010, Korea. E-mail: april31kjh@naver.com

Copyright () 2015 The Korean Society for Aesthetic Plastic Surgery.

This is an Open Access article distributed under the terms of the Creative Commons Attribution Non-Commercial License (http://creativecommons.org/licenses/by-nc/3.0/) which permits unrestricted non-commercial use, distribution, and reproduction in any medium, provided the original work is properly cited. $\quad w w w . e-a a p s . o r g$
The 6th, 7th, and 8th ribs are most often selected for harvest [12]. However, the use of these costal cartilages has some disadvantages, including donor-site morbidities such as vessel damage or iatrogenic pneumothorax during the harvest, chest wall deformities, persistent postoperative pain, additional scarring in the chest wall, and potential warping. Various costal cartilage harvesting and carving techniques have been introduced to overcome the aforementioned disadvantages. With such efforts, both donor-site morbidity and warping have decreased significantly [13-22].

However, costal cartilage harvesting still requires time and careful attention, and disadvantages such as donor-site morbidity and warping are not completely overcome. Therefore, as long as dorsal augmentation with costal cartilage is not considered, we planned to use the 10th costal cartilage to achieve good results with minimal harvesting of the cartilage.

We would like to introduce the use of the 10th costal cartilage for nasal tip-plasty. 


\section{METHODS}

Of the patients who underwent rhinoplasty using the 10th costal cartilage between November 2008 and December 2012, 64 patients who could be observed and followed for longer than 6 months were enrolled in this study. The indications for using the 10th costal cartilage were as follows: severe caudal nasal deviation, secondary cleft lip nasal deformity, and secondary rhinoplasty with difficulty of using the septal and auricular cartilages.

The medical charts and operative records were reviewed retrospectively to evaluate the use of the cartilage, donor-site morbidity, and postoperative outcomes, including complications. An independent evaluator assessed donor-site scarring using a five-point scale 6 months after the surgery, based on pigmentation and pliability (1 = very poor, $2=$ poor, $3=$ neutral, $4=$ good, and $5=$ very good). The study conformed to the Declaration of Helsinki. Written consent was obtained from each patient for both the surgery and publication of the photographs of the results.

\section{Surgical procedure Marking}

The location of the 10th costal cartilage was marked using manual palpation while the patient was in the supine position. Whereas the second rib is normally used to confirm the location of the 5th and 6th ribs, the location of the 10th rib, which is connected to the costal arch, can be confirmed after verifying the location of the 11th rib, which is a floating rib, through manual palpation. When the soft tissue is too thick and manual palpation becomes difficult, the 10th rib is located at the costal arch, where the hypothetical lines from the anterior superior iliac spine and the umbilicus meet at a 90-degree angle (Fig. 1). A $1 \mathrm{~cm}$ to $1.5 \mathrm{~cm}$ incision was made directly over the 10th costal cartilage to facilitate dissection.

\section{Harvesting of the 10th costal cartilage}

All surgeries were performed under conscious sedation. The anesthetic solution consisted of $1 \%$ lidocaine with 1:200,000 epinephrine. The local anesthetic solution was administered at the incision line, and skin incision was made using a No. 15 scalpel. The subcutaneous tissue was dissected using electrocautery, and the external oblique abdominis muscle (EOAM) was exposed. A Senn retractor was used to clear the visual field, and the underlying rib was manually palpated to check the location. The EOAM was dissected to expose the costal cartilage.

Once the costal cartilage was exposed, an incision was made through the anterior surface of the perichondrium using a No. 15 scalpel. The perichondrium was dissected using a freer septum elevator. Because the medial portion of the 10th costal cartilage is thinner, the undersurface of the 10th cartilage is easy to detach from
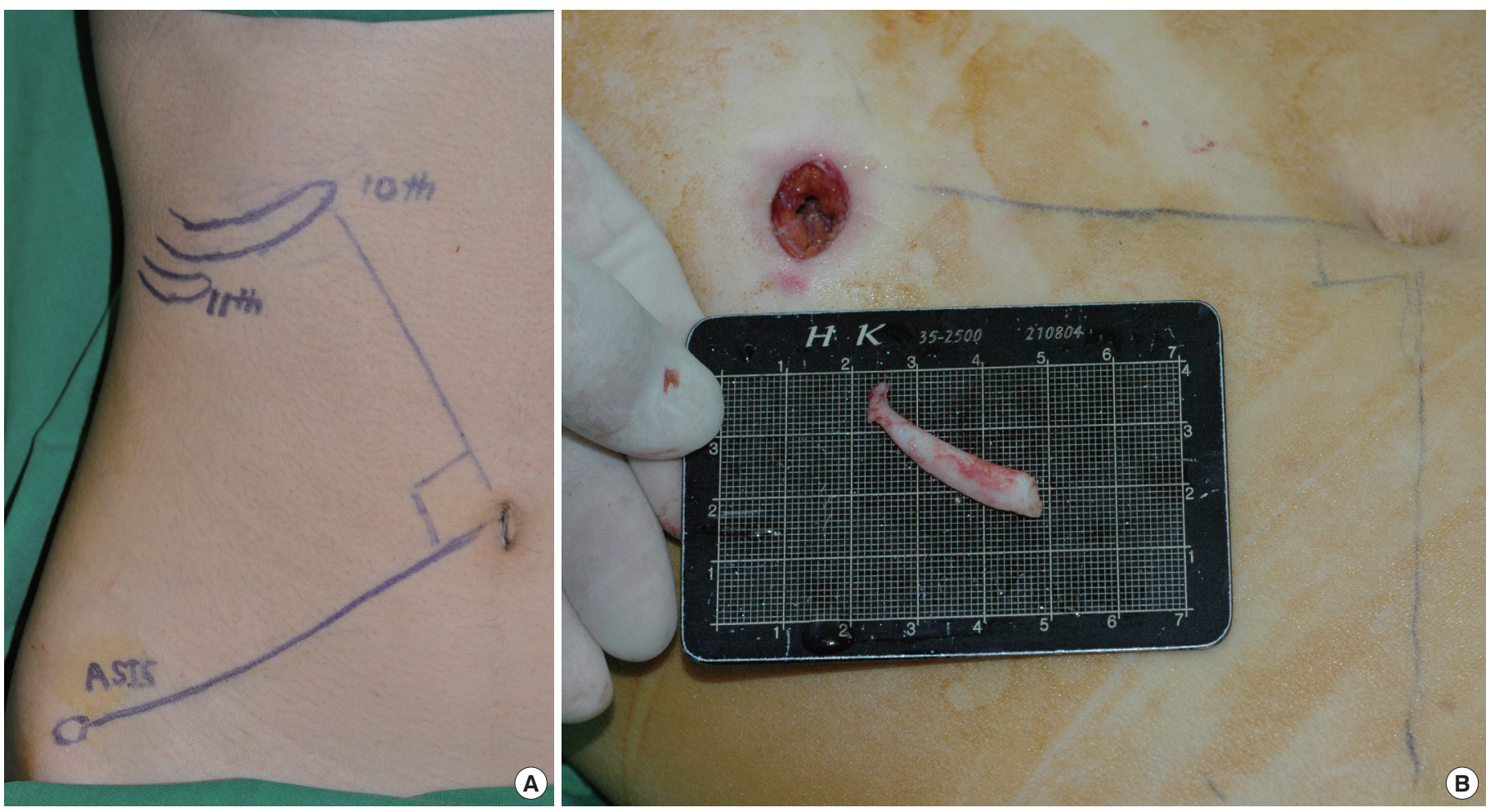

Fig. 1. (A) Design of the costal cartilage donor-site. The 10th costal cartilage is located close to the point at which the anterior superior iliac spine and the umbilicus and costal arch meet at a 90-degree angle. (B) The cartilage graft harvested from the right 10 th rib. 
the posterior perichondrium without causing any damage, and using only an elevator. The fibrotic tissue connected to the 9th cartilage was detached to separate the 10th cartilage. The cartilage was fixed using the Brown-Adson forceps, and the perichondrium was circumferentially dissected in the lateral direction of the cartilage, using an elevator. The required size of the cartilage was exposed, and a cartilaginous cut was made (see video, Supplemental Digital Content 1). After the meticulous bleeding control was completed, separated muscles were approximated and the wound was closed layer by layer. No drain was inserted and a compressive dressing was applied for 3 to 4 days.

\section{Use of the 10th costal cartilage in tip-plasty}

The harvested cartilage was used as a columellar strut to support the tripod structure. For rhinoplasty cases that require strong struts, such as a severe caudal nasal deviation or secondary cleft lip nasal deformity, the cartilage was used as a fixed-type columellar strut with a Kirschner wire (Fig. 2); for secondary rhinoplasty that lacks septal and conchal cartilages, the cartilage was mainly used as the floating-type columellar strut. The 10th costal cartilage was used as
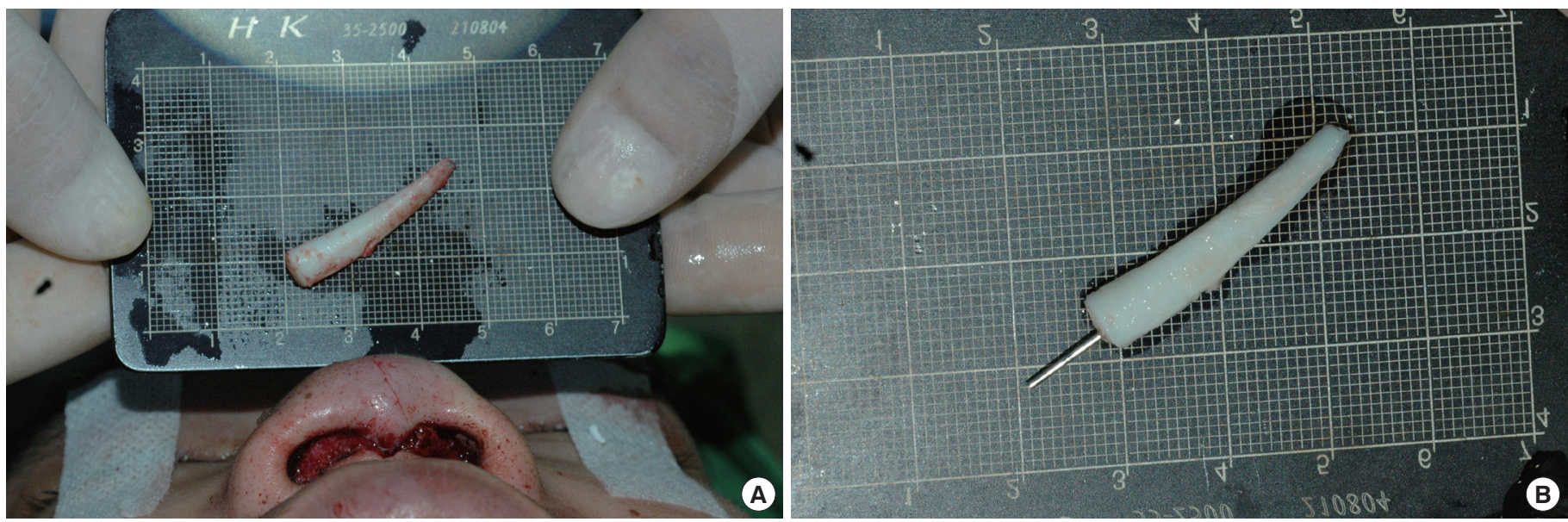

Fig. 2. (A) The cartilage graft harvested from the 10th rib. (B) The Kirschner wired costal cartilage graft with the simply trimmed 10 th costal cartilage just prior to insertion.
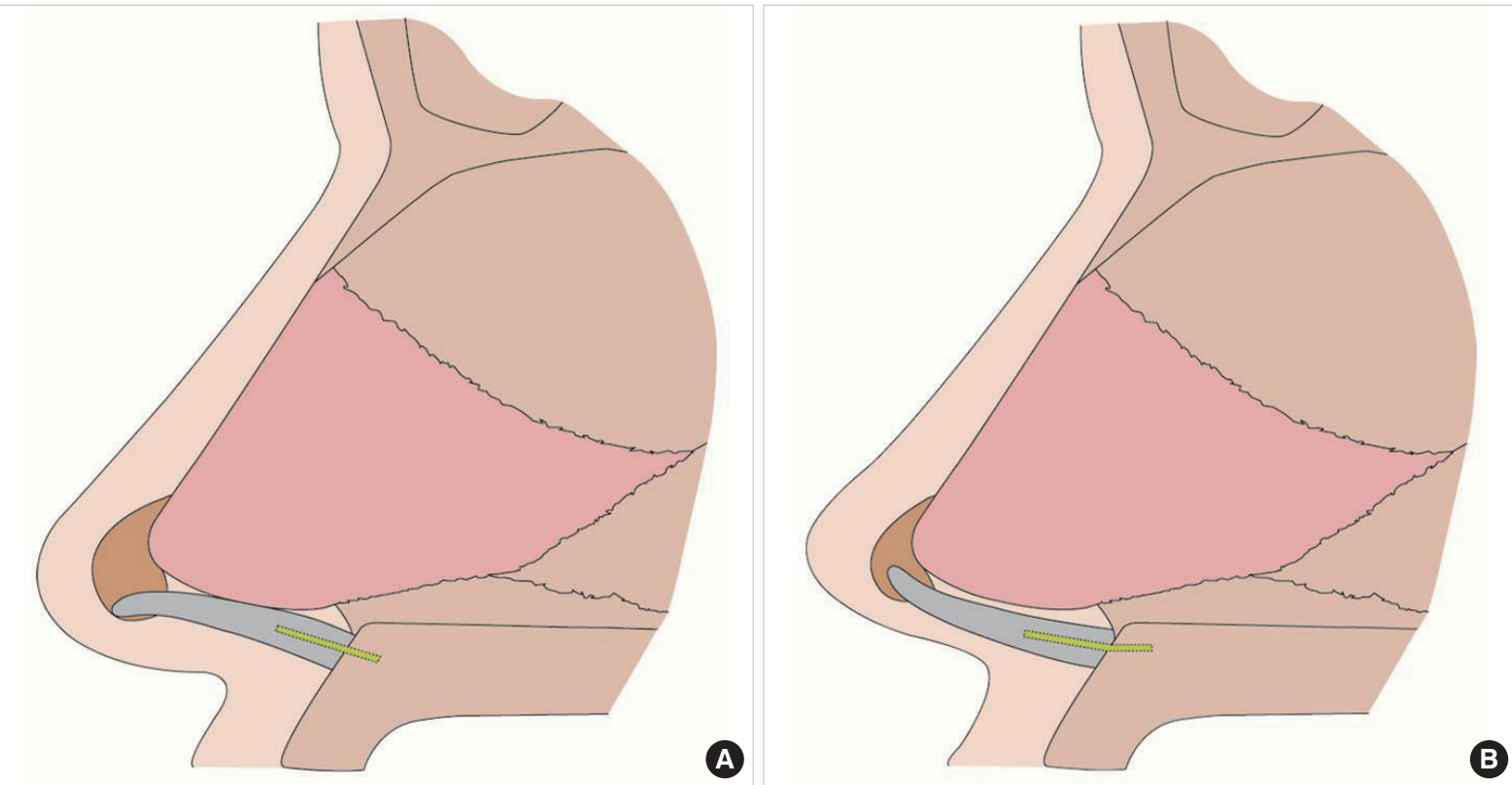

Fig. 3. Schematic presentation of applying the natural curvature of the 10th costal cartilage according to its use as a strut. (A) The 10th costal cartilage was placed to curve downward when nasal tip lengthening was mainly needed and (B) to curve upward when tip projection was the main purpose. 
a columellar strut without carving, or after carving on both sides based on the balanced cross-section principle in case the cartilage was too thick. Based on the purpose of the strut, the natural curvature of the 10th cartilage was used as is (Fig. 3).

When tip grafts were required, a cartilage crusher was used to avoid the postoperative graft visibility. For nasal dorsal augmentation, a boat-shaped silicone implant was mainly used.

\section{RESULTS}

Of the 64 patients, 13 were men and 51 were women. The mean age of the patients was 32.7 years (range, 21-53 years), and their mean follow up period was 9 months (range, 6-27 months). Seven (11\%) of the patients had caudal nasal deviation; $5(8 \%)$ patients had secondary cleft lip nasal deformity; and 52 (81\%) patients had secondary rhinoplasty. In most cases, the length of the incision was $1 \mathrm{~cm}$ to $1.5 \mathrm{~cm}$. The mean time taken to harvest the cartilage was 18 minutes (range, 13-27 minutes), and the mean length of the harvested cartilage was $2.4 \mathrm{~cm}$ (range, 1.5-3.8 cm). No donor-site complications, such as blood vessel damage or peritoneal entry, were observed. Fifteen harvested cartilages (23\%) were used as fixed columellar struts, and 49 cartilages (77\%) were used as floating columellar struts. The cartilage carving process was unnecessary in 38 cases (59\%). Cartilages were used for tip onlay grafts in 51 cases; 2 cases required alar contour grafts for secondary cleft lip nasal deformity.

Most patients had satisfactory aesthetic results. No infections or severe cases of warping related to the graft were observed (Fig. 4-6). Although three patients underwent revision, it was not because of a graft-related problem such as columellar deviation, but rather due to supra-tip depression (1 case) and demarcation of the tip graft (2 cases).

In the objective evaluation of the donor-site scars, 16 cases (25\%) showed very good quality scars; 31 cases ( $48 \%$ ) showed good quality scars; 13 cases (20\%) showed neutral scars; 1 case (2\%) showed a poor quality scar; and 3 cases (5\%) showed poor quality scars.

Although intralesional steroid injections were used in two cases of poor quality scars because they were hypertrophic at the donorsite, operative revision was unnecessary.

\section{DISCUSSION}

The aim of this study was to evaluate the efficacy and safety of the 10th costal cartilage for rhinoplasty. Primarily, the difference between conventionally used costal cartilage and the 10th costal cartilage should be understood. Harvesting of the 5th or 6th costal cartilage can minimize the visibility of the incision, because the incision is located in the inframammary fold in female patients [6], and the cartilage is straighter. However, there are potential risks of pneumothorax and blood vessel damage, as the internal thoracic vessels pass deep to the costal cartilages [23].

The 7th and 8th costal cartilages are also often used, and the fact that large amounts of cartilage can be harvested with convenient accessibility is an advantage. As the diaphragm is situated inferior to the cartilage, pneumothorax or blood vessel injuries are rare [23]. However, the relatively thick rectus abdominis muscle (RAM) drapes downward over the cartilages, and dissection of greater amounts of muscles is required. Accordingly, a larger incision is also required,
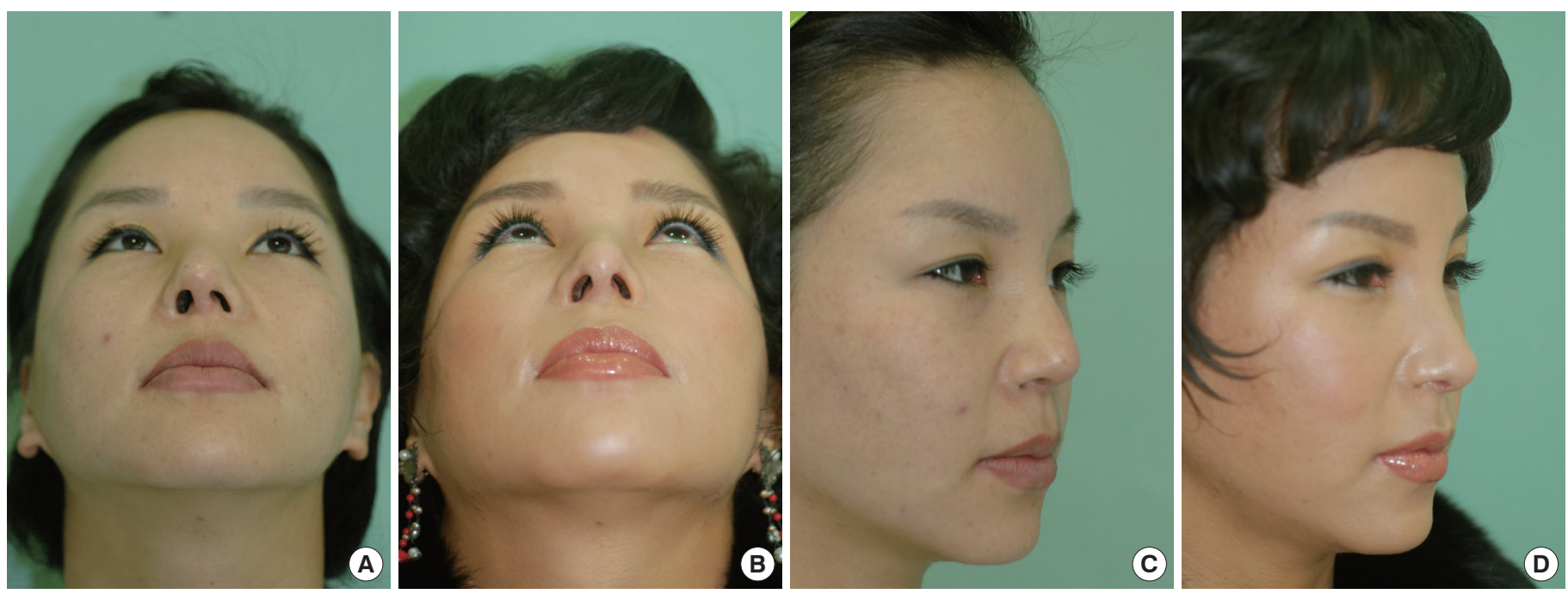

Fig. 4. A 42-year-old secondary patient presented with caudal nasal deviation. She had undergone a previous rhinoplasty but still exhibited the caudal nasal deviation. She underwent an open approach, including a fixed columellar strut using the 10th costal cartilage and Kirschner wire for correction of the caudal nasal deviation. (A) Preoperative inferior view. (B) Postoperative inferior view at 9 months. (C) Preoperative oblique view. (D) Postoperative oblique view at 9 months. 
which increases donor-site morbidity and postoperative pain [24].

The 11th costal cartilage is rarely used for nasal dorsum reconstruction. While the floating rib is flexible, the 11th costal cartilage is harvested only through a small incision. Additionally, the scar caused by the skin incision is of a relatively good quality, as it is located away from the sternal region [25]. However, accurate palpation to locate the 11th costal cartilage is difficult in patients with thick soft tissue, and the amount of cartilage that can be harvested is limited. In our experience, cartilage damage during harvesting often occurs with the 11th costal cartilage because it is very thin and fragile.

The 10th costal cartilage, which was used in this study, is situated above the abdominal cavity of the inferior thorax and above the diaphragm and transverse abdominis muscle. As such, there are lower risks of pneumothorax or peritoneal entry. Moreover, such costal cartilage is easily found because it is connected to the costal
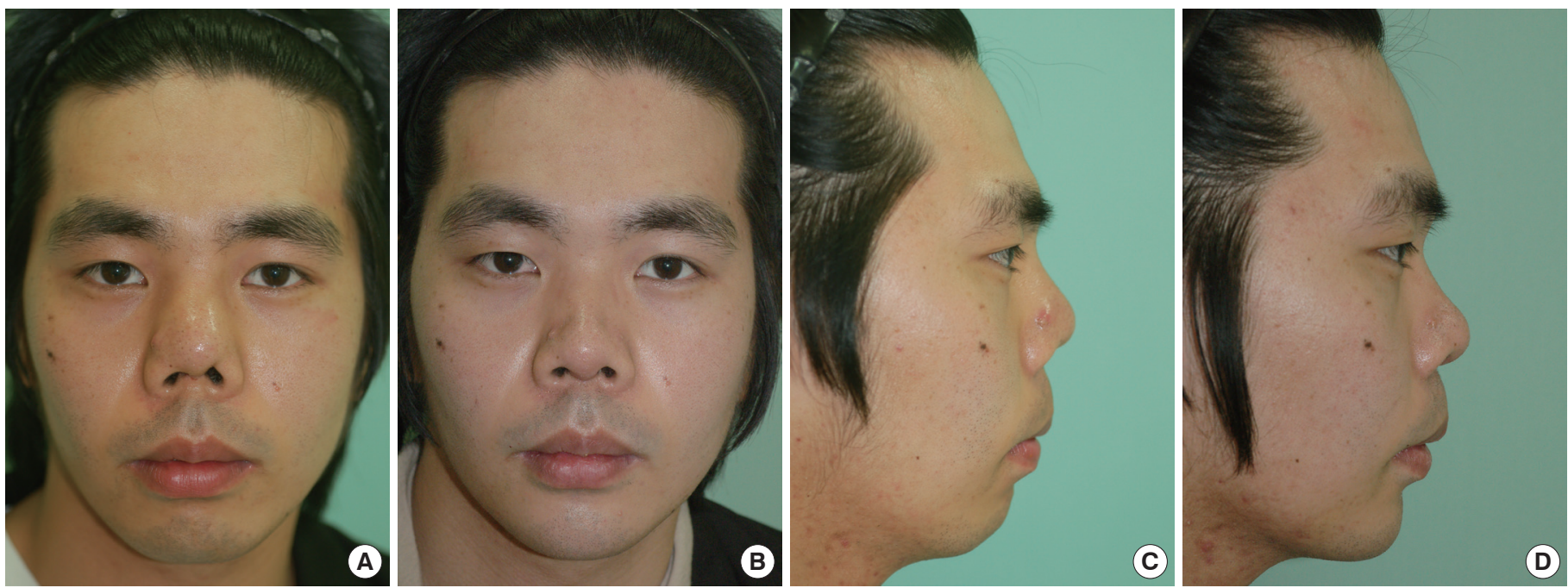

Fig. 5. A 29-year-old secondary patient presented with a contracted nose. He had undergone an augmentation rhinoplasty using a Gore-Tex implant, and presented with the destruction of nasal tip architecture and exposed implant through the vestibular mucosa. The Gore-Tex implant and hypertrophic capsule were removed, sufficient caudal rotation of alar cartilages was allowed, and the fixed columellar strut using the 10th costal cartilage and Kirschner wire was used to rebuild the nasal tip. He underwent dorsal augmentation with a boat-shaped silicone implant, and chin augmentation was performed simultaneously. (A) Preoperative front view. (B) Postoperative front view at 6 months. (C) Preoperative lateral view. (D)Postoperative lateral view at 6 months.
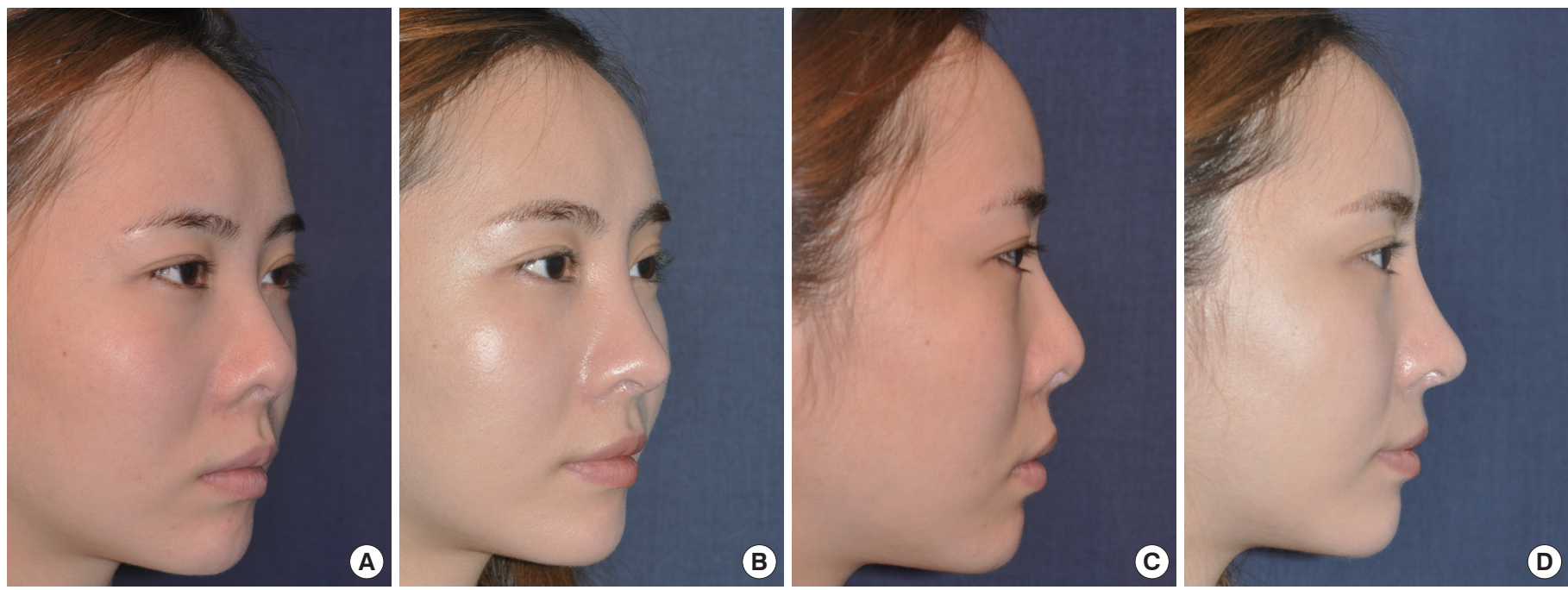

Fig. 6. A 27-year-old secondary patient presented with a contracted nose. In two prior operations, the septal and auricular cartilages were used. Free movement of the tripod structure was achieved by the complete dissection of the scar tissue and support structure that was firmly holding the tip. The nasal tip was reconstructed with a floating columellar strut, using the 10th costal cartilage, and a tip onlay graft. (A) Preoperative oblique view. (B) Postoperative oblique view at 6 months. (C) Preoperative lateral view. (D) Postoperative lateral view at 6 months. 


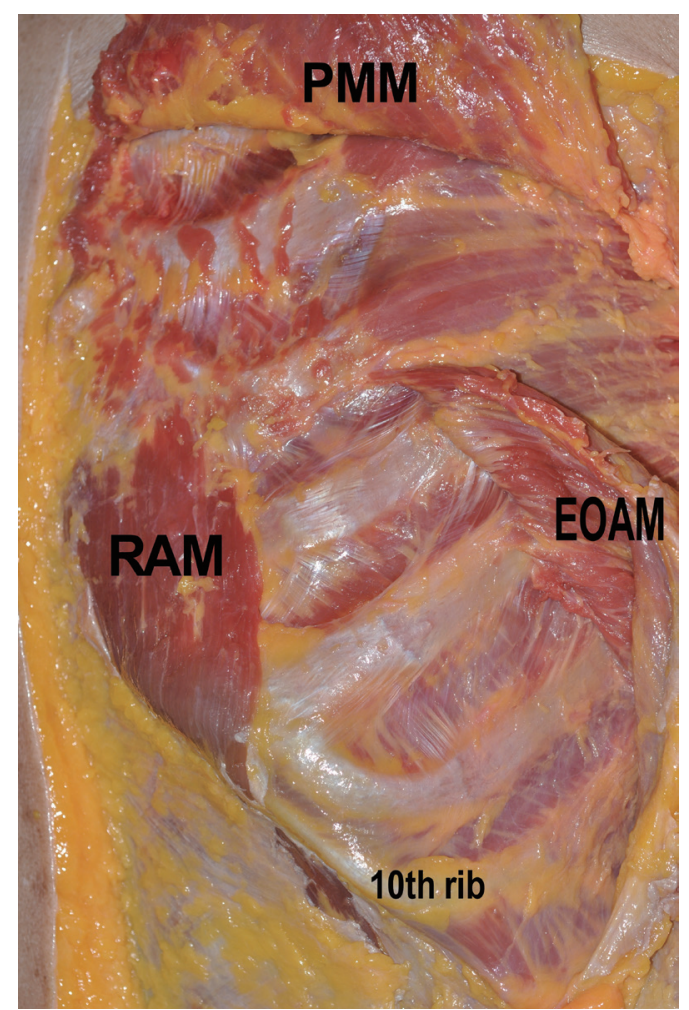

Fig. 7. A cadaveric study view shows the location relation between the rectus abdominis muscle (RAM) and the 10th rib. As the position of the 10th costal cartilage is more lateral than the RAM, the 10th costal cartilage can be harvested without dissection of the RAM. EOAM, external oblique abdominis muscle; PMM, pectoralis major muscle; RAM, rectus abdominis muscle.

arch, and it can be harvested with the 9th costal cartilage if needed. Christophel and Hilger [17] used an osseocartilaginous rib graft for dorsal nasal reconstruction, for which they used mainly the distal 10th rib or the floating 11th rib due to their thinness and accessibility. Moreover, since the 10th costal cartilage is located lateral to the RAM, the dissection of the relatively thick RAM is not required during the cartilage harvesting (Fig. 7). Accordingly, donorsite morbidity and postoperative pain decrease.

Because the autologous costal cartilage is a dynamic and living piece of tissue, warping has been reported after carving [26,27]. Although many studies have been conducted to resolve the warping problem [13,17-21,26], avoiding carving is considered the most desirable solution. In this study, no severe warping was observed; this could be attributed to our use of the 10th costal cartilage, which is relatively thin at $10.6 \mathrm{~mm}$ (right) and $10.5 \mathrm{~mm}$ (left), according to the cadaveric analyses [23]. The 10th costal cartilage thins towards its medial portion. Because its natural curvature is used for the strut, carving can be minimized. In this study, 38 10th costal cartilages (59\%) were used as struts without carving. In the dorsal augmentation cases for saddle nose deformities, Al-Qattan [28]

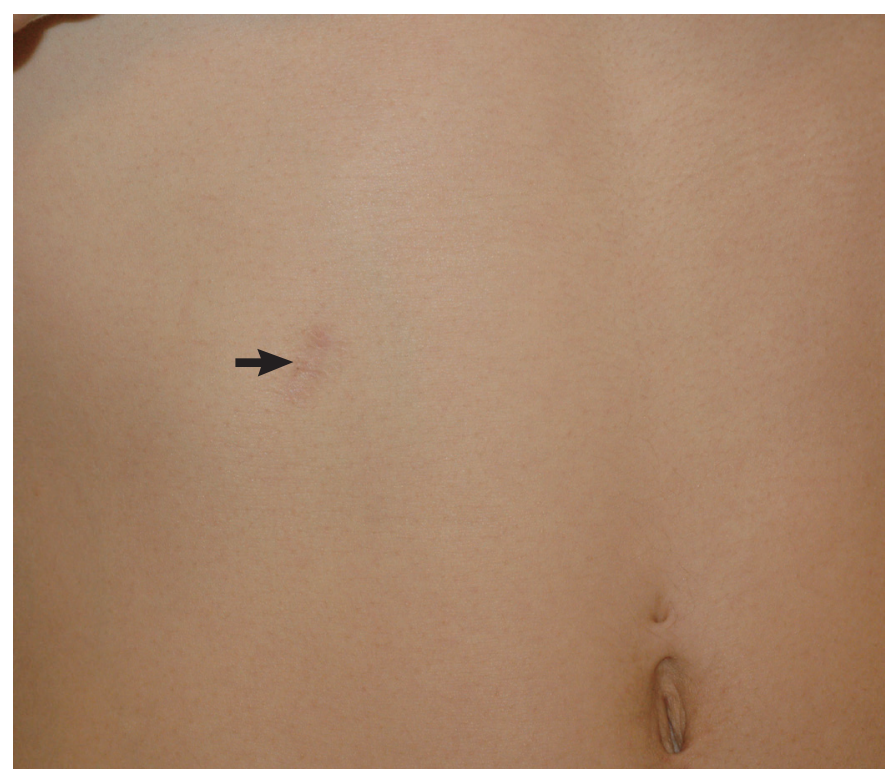

Fig. 8. Donor-site scar after 24 months in a patient who had undergone the harvesting of the 10th costal cartilage, demonstrating relatively good quality.

used the 9th or 10th costal cartilage without carving, after its inferior border was rotated by 90 degrees to make a convex dorsum. No warping was reported during the long-term follow up. This report also showed that avoiding or minimizing carving could shorten the surgical time and reduce operator fatigue.

The donor-site scar is another important issue. In particular, a hypertrophic scar is often observed in Asians or Africans. However, extremely small incisions for harvesting costal cartilage may result in fatal donor-site morbidity. Harvesting the 10th costal cartilage minimizes donor-site scarring based on two factors: the 10th costal cartilage is relatively far from the sternal region, which is known to cause poor quality scars, and the 10th costal cartilage does not lie over the internal thoracic vessels or pleura, which allows for a smaller incision [25] (Fig. 8).

Autologous costal cartilage can be used for rhinoplasty in various forms (as a cantilever, tongue in groove, spreader, columellar strut, and septal extension graft). As Toriumi and Swartout [29] reported, when tip lengthening is required, the use of septal extension or spreader graft forms can result in good nasal tip lengthening. However, we do not think that a permanent fixation force can be achieved with an extension graft; instead, we believe that a more important role for the graft is to serve as a strut that is maintained until the formation of a stable biologic scar can be achieved with minimum tension [30], rather than as a permanent strut [5]. Therefore, we believe that good results can be achieved even with the minimal use of the costal cartilage, when a sufficiently released tripod structure is supported by a columellar strut and the mobility of the soft tissue skin envelope is improved. Although the septal and au- 
ricular cartilages are considered primary types of cartilage graft material for rhinoplasty, the 10th costal cartilage could also be considered primary graft material in patients who have experienced trauma that increases the risk of septal cartilage damage, who have undergone prior rhinoplasty using the septal and conchal cartilages, or who have a hypertrophic scar or a keloid in the auricular area.

\section{CONCLUSION}

We conclude that the harvesting and usage of the 10th costal cartilage is a safe and effective method for rhinoplasty. Hence, the 10th costal cartilage can be considered an option for an autologous costal cartilage graft in rhinoplasty. It can also be considered primary graft material for selective rhinoplasty cases, as is septal or auricular cartilage.

\section{APPENDIX}

Supplemental Digital Content 1. This video shows the harvest of the 10th costal cartilage.

\section{PATIENT CONSENT}

Patients provided written consent for the use of their images.

\section{REFERENCES}

1. Gunter JP, Rohrich RJ. Lengthening the aesthetically short nose. Plast Reconstr Surg 1989;83:793-800.

2. Gunter JP, Rohrich RJ. Augmentation rhinoplasty: dorsal onlay grafting using shaped autogenous septal cartilage. Plast Reconstr Surg 1990; 86:39-45.

3. Jang YJ, Yu MS. Rhinoplasty for the Asian nose. Facial Plast Surg 2010; 26:93-101.

4. Kamer FM. Lengthening the short nose. Ann Plast Surg 1980;4:281-5.

5. Kim JH, Song JW, Park SW, et al. Effective septal extension graft for asian rhinoplasty. Arch Plast Surg 2014;41:3-11.

6. Marin VP, Landecker A, Gunter JP. Harvesting rib cartilage grafts for secondary rhinoplasty. Plast Reconstr Surg 2008;121:1442-8.

7. Cochran CS, Gunter JP. Secondary rhinoplasty and the use of autogenous rib cartilage grafts. Clin Plast Surg 2010;37:371-82.

8. Defatta RJ, Williams EF, 3rd. The decision process in choosing costal cartilage for use in revision rhinoplasty. Facial Plast Surg 2008;24:365-71.

9. Hafezi F, Naghibzadeh B, Ashtiani AK, et al. Correction of cleft lip nose deformity with rib cartilage. Aesthet Surg J 2013;33:662-73.

10. Park JH, Jin HR. Use of autologous costal cartilage in Asian rhinoplasty. Plast Reconstr Surg 2012;130:1338-48.

11. Sherris DA, Kern EB. The versatile autogenous rib graft in septorhino- plasty. Am J Rhinol 1998;12:221-7.

12. Toriumi DM, Patel AB, DeRosa J. Correcting the short nose in revision rhinoplasty. Facial Plast Surg Clin North Am 2006;14:343-55.

13. Daniel RK. Rhinoplasty and rib grafts: evolving a flexible operative technique. Plast Reconstr Surg 1994;94:597-609.

14. Ağaoğlu G, Erol OO. In situ split costal cartilage graft harvesting through a small incision using a gouge. Plast Reconstr Surg 2000;106:932-5.

15. Boyaci Z, Çelik Ö, Ateşpare A, et al. Conservative costal cartilage harvest for revision septorhinoplasty. J Craniofac Surg 2013;24:975-7.

16. Ching WC, Hsiao YC. Transumbilical endoscopic costal cartilage harvesting: a new technique. Ann Plast Surg 2014;72:423-7.

17. Christophel JJ, Hilger PA. Osseocartilaginous rib graft rhinoplasty: a stable, predictable technique for major dorsal reconstruction. Arch Facial Plast Surg 2011;13:78-83.

18. Clark JM, Cook TA. Immediate reconstruction of extruded alloplastic nasal implants with irradiated homograft costal cartilage. Laryngoscope 2002;112:968-74.

19. Foulad A, Hamamoto A, Manuel C, et al. Precise and rapid costal cartilage graft sectioning using a novel device: clinical application. JAMA Facial Plast Surg 2014;16:107-12.

20. Gunter JP, Clark CP, Friedman RM. Internal stabilization of autogenous rib cartilage grafts in rhinoplasty: a barrier to cartilage warping. Plast Reconstr Surg 1997;100:161-9.

21. Kim DW, Shah AR, Toriumi DM. Concentric and eccentric carved costal cartilage: a comparison of warping. Arch Facial Plast Surg 2006; 8:42-6.

22. Kobayashi S, Yoza S, Takada H, et al. Endoscope-assisted rib cartilage harvesting. Ann Plast Surg 1995;35:571-5.

23. Jung $\mathrm{DH}$, Choi $\mathrm{SH}$, Moon HJ, et al. A cadaveric analysis of the ideal costal cartilage graft for Asian rhinoplasty. Plast Reconstr Surg 2004; 114:545-50.

24. Cakmak O, Ergin T. The versatile autogenous costal cartilage graft in septorhinoplasty. Arch Facial Plast Surg 2002;4:172-6.

25. Gentile P, Cervelli V. Nasal dorsum reconstruction with 11th rib cartilage and auricular cartilage grafts. Ann Plast Surg 2009;62:63-6.

26. Farkas JP, Lee MR, Lakianhi C, et al. Effects of carving plane, level of harvest, and oppositional suturing techniques on costal cartilage warping. Plast Reconstr Surg 2013;132:319-25.

27. Toriumi DM. Discussion: Use of autologous costal cartilage in Asian rhinoplasty. Plast Reconstr Surg 2012;130:1349-50.

28. Al-Qattan MM. Augmentation of the nasal dorsum with autogenous costal cartilage using the "edge-on" technique. Ann Plast Surg 2007;59: 642-4.

29. Toriumi DM, Swartout B. Asian rhinoplasty. Facial Plast Surg Clin North Am 2007;15:293-307, v.

30. Tebbetts JB. Primary rhinoplasty : a new approach to the logic and the techniques. St. Louis, MO: Mosby; 1998. 